\title{
Experimental Hematology
}

National Cancer Institute

\section{Source}

National Cancer Institute. Experimental Hematology. NCI Thesaurus. Code C17819.

Basic research in the field of hematology. 